\title{
How Does Behavioural Activation Work? A Systematic Review of the Evidence on Potential Mediators
}

\author{
Noortje P. Janssen ${ }^{a, b, c}$ Gert-Jan Hendriks ${ }^{a, c, f} \quad$ Céline T. Baranellib \\ Peter Lucassen $^{b}$ Richard Oude Voshaare Jan Spijker ${ }^{a, c, f}$ Marcus J.H. Huibers ${ }^{d}$ \\ a Behavioural Science Institute, Radboud University, Nijmegen, The Netherlands; ${ }^{b}$ Department of Primary and \\ Community Care, Research Institute of Health Sciences, Radboud University Medical Centre Nijmegen, Nijmegen, \\ The Netherlands; ' Institute for Integrated Mental Health Care "Pro Persona," Nijmegen, The Netherlands; \\ dDepartment of Clinical Psychology, VU University, Amsterdam, The Netherlands; 'University Medical Center \\ Groningen, Interdisciplinary Center for Psychopathology of Emotion Regulation (ICPE), University of Groningen, \\ Groningen, The Netherlands; ${ }^{f}$ Department of Psychiatry, Radboud University Medical Centre Nijmegen, Nijmegen, \\ The Netherlands
}

\section{Keywords}

Behavioural activation - Depression - Mechanisms of change $\cdot$ Mediation $\cdot$ Quality assessment

\section{Abstract \\ Introduction: Behavioural activation is an effective treat- ment for depression, but little is known about its working mechanisms. Theoretically, its effect is thought to rely on an interplay between activation and environmental reward. Objective: The present systematic review examines the me- diators of behavioural activation for depression. Methods: A systematic literature search without time restrictions in Medline, EMBASE, PsycINFO, The Cochrane Library, and CINAHL resulted in 14 relevant controlled and uncontrolled prospective treatment studies that also performed formal mediation analyses to investigate their working mecha- nisms. After categorising the mediators investigated, we sys- tematically compared the studies' methodological quality and performed a narrative synthesis of the findings. Results: Most studies focused on activation or environmental re- ward, with 21 different mediators being investigated using}

karger@karger.com www.karger.com/pps

Karger $\frac{1}{6}$

GOPEN ACCESS
(C) 2020 The Author(s)

Published by S. Karger AG, Basel

This article is licensed under the Creative Commons AttributionNonCommercial-NoDerivatives 4.0 International License (CC BYNC-ND) (http://www.karger.com/Services/OpenAccessLicense). Usage and distribution for commercial purposes as well as any distribution of modified material requires written permission. questionnaires that focused on psychological processes or beliefs. The evidence for both activation and environmental reward as mediators was weak. Conclusions: Non-significant results, poor methodological quality of some of the studies, and differences in questionnaires employed precluded any firm conclusions as to the significance of any of the mediators. Future research should exploit knowledge from fundamental research, such as reward motivation from neurobiology. Furthermore, the use of experience sampling methods and idiographic analyses in bigger samples is recommended to investigate potential causal pathways in individual patients.

(c) 2020 The Author(s) Published by S. Karger AG, Basel

\section{Introduction}

Depression is a common mental disorder and is associated with a substantial burden for patients and those around them [1-3]. Currently, pharmacotherapy, cognitive behavioural therapy, and interpersonal therapy are the most frequently used evidence-based psychological 
treatments for depression, but research suggests that behavioural activation (BA) is equally effective [4-9] while it is less costly and complex than other psychotherapies $[10,11]$.

BA is well structured and aims to increase adaptive, pleasurable activities, and decrease maladaptive, depression-maintaining activities [12]. The intervention is based on the behavioural models of Lewinsohn that assume that depressed individuals obtain less positive reinforcement than other individuals because they engage less frequently in pleasant activities and enjoy activities less $[7,13]$. Lewinsohn [14] hypothesised that having patients engage in pleasant activities will improve their depressed mood. In BA, by monitoring their mood and activities, patients learn to understand the connection between what they do and how they feel, and use this knowledge to schedule activities that create an atmosphere of positive reinforcement and to solve problems that prevent them from doing so [12]. The empirical evidence for the effectiveness of BA for adult depression is extensive and convincing $[7,8,15]$, and that for depression in young people [16] and older adults [17] is also growing. However, as with other evidence-based psychotherapies, less is known about how BA works and which processes make the treatment effective [18].

Early observational studies provided some correlational evidence on the theoretical background of BA, showing that pleasant activities are associated with fluctuations in depressed mood $[19,20]$, with depressed adults experiencing the same events as less enjoyable than non-depressed adults, explaining why patients experience less pleasure [13]. More recent studies showed that mood is indeed associated with increased activity levels [21-23] and that brain activity in regions associated with approach-related behaviour and those associated with reward processing was lower than it was in non-depressed adults $[24,25]$. Although the original research on which BA is founded is important for understanding the theory and the various processes that might be involved [12], evidence on the causality of the presumed associations is still lacking. We know that BA works, but to explain how it works we need to focus on mechanisms of change, i.e., which processes cause which outcomes and how $[18,26,27]$.

According to Kazdin $[26,28]$ an important step in understanding mechanisms of change is to identify and investigate possible mediators, i.e., those variables that statistically explain the relationship between a dependent and an independent variable. Although a mediator is not necessarily a mechanism - it does not always explain the actual processes through which treatment leads to change - it can point towards possible mechanisms that are accountable for outcome [26, 29]. Kazdin [26] formulated several requirements for research on mechanisms of change using mediators, which can be found in the online supplementary material of this paper (for all online suppl. material, see www.karger.com/doi/10.1159/000509820). The more these requirements are fulfilled, the stronger the evidence that a certain mediator explains the relationship between treatment and outcome. By identifying mediators, we can start to explicate the actual underlying mechanisms that these mediators represent, and when we know how a treatment works, we can refine protocols and increase the generalisability of treatment effects from research to clinical practice $[26,27]$.

Since a comprehensive overview of the mediators involved in BA is lacking, in the present review we systematically identify and summarise the mediators that have been studied to evaluate the strength of evidence for their roles in the putative mechanism of BA, and discuss how this knowledge can shape future studies.

\section{Methods}

Search Procedure and Eligibility

This review was conducted in accordance with the Preferred Reporting Items for Systematic Reviews and Meta-Analyses (PRISMA) guidelines [30]. Methods and inclusion criteria were included in a protocol (PROSPERO registration: CRD42019119696) in advance of the study. Due to the pre-estimated scarcity of articles about the mediators of BA as well as the heterogeneity of study populations, methodologies, and outcome measures, we decided to refrain from doing a meta-analysis, because bundling such heterogeneous information in one analysis has no added value for both researchers as clinicians in understanding the working mechanisms of BA [31, 32].

Only studies that had performed a formal test of mediation of processes [33] and outcome of BA were included that hence reported statistical data or inferred indirect effects of mediators on the relationship between treatment and depression outcome. No restrictions on language, publication date, or publication status were imposed. Studies could include patients of any age with a depressive disorder, dysthymia, or depressive symptoms, be controlled or uncontrolled, or compare any form of BA therapy or traditional behaviour therapy for depression to any comparator. All settings and forms of delivery were allowed, including inpatient and outpatient populations, individual and group sessions, as well as traditional face-to-face and self-help (internet or bibliotherapy) interventions. Studies on patients with bipolar disorder were excluded.

Our primary outcome was the relationship between BA treatment, its potential mediators, and depression outcome. Depression outcome could be measured using any kind of quantitative depression-specific self-rated or clinician-rated scale.
Janssen/Hendriks/Baranelli/Lucassen/ Oude Voshaar/Spijker/Huibers 
Abstracts of papers were selected from Medline, EMBASE, PsycINFO, The Cochrane Library, and CINAHL from inception to March 2, 2020 using variations of the following search string: (Exp Major depression/ OR "depression (emotion)"/ OR Seasonal Affective Disorder/ OR Atypical depression/ OR premenstrual dysphoric disorder/ OR (depress* OR dysthymi?).ti,ab,id.) AND (Behavior Therapy/ OR ((behavio* ADJ2 (therap* OR psychotherap* OR activation OR treatment? OR intervention?)) OR activity schedul*).ti,ab,id) AND (mechanism? OR process* OR change OR mediat* OR action?).ti,ab,id. The search string was peer reviewed with the PRESS checklist [34] by 2 independent information specialists of university medical centres in the Netherlands. In addition to the database search, we checked reference lists for additional relevant publications and asked several experts on BA to suggest papers related to the topic.

\section{Study Selection and Data Collection}

Titles and abstracts for all identified studies were screened for eligibility by N.P.J. and C.T.B. using Rayyan [35], and studies not meeting any of the abovementioned inclusion criteria were excluded. Of the remaining studies, the full-text papers were independently screened (N.P.J. and C.T.B.). Decisions were based on a detailed coding manual with predefined selection criteria. In case of disagreement, the reviewers discussed the discrepancy and contacted a third reviewer (M.H.), if needed, to reach a consensus. Next, the type and results of the mediation analyses were extracted, and the sex, age, and diagnosis of the patient samples, and type of BA and control group (both active treatment arm and waiting list) were recorded.

\section{Quality Assessment and Synthesis of Results}

While quality assessment of mediational studies is highly important, validated tools to test the risk of bias of randomised controlled trials (RCTs), such as the Cochrane risk of bias tool, do not adequately measure the quality of mediational studies. We therefore decided to construct a dedicated quality checklist based on Kazdin's [26] requirements outlined above and the work of Cooper et al. [36] to help us rate the quality of the analyses employed to measure associations, temporal precedence, specificity, study design, power and missing data, and representativeness of the treatment, and thus determine the strength of the evidence of the mediators reported on. Each requirement was rated on a 3-point Likert scale, with 0 indicating low quality and 2 high quality, yielding a maximum score of 12 . A detailed description of our quality assessment strategy can be found in the online supplementary material on page 2 .

The quality assessment was performed independently by N.P.J. and G.H., and again, in case of disagreement, they discussed the discrepancy and, if needed, involved a third reviewer (M.H.) to reach a consensus. To determine the actual strength of the evidence of the mediators, apart from the study quality, we considered whether mediators were consistently replicated across the included studies using adequate study designs. By reviewing all studies that performed a mediation analysis regardless of quality, we sought to provide as complete an overview of all mediators investigated in the context of BA as possible. As to the strength of evidence, there is no mention of a fixed cut-off threshold in the literature to divide high- and low-quality mediation studies, which is why we, in our narrative synthesis, gave more weight to the results of high-quality studies than to the results of studies with a lower quality rating.

How Does Behavioural Activation Work?

\section{Results}

\section{Study Selection and Characteristics}

The database searches yielded 17,544 hits. Questioning BA experts yielded an additional 8 titles. After removing duplicates, a total of 11,052 titles and abstracts were screened for eligibility. Of these, 10,825 did not meet the inclusion criteria, leaving 227 titles for full-text screening. From these, we could include 14 papers that fulfilled the inclusion criteria. The reasons for exclusion are listed in online supplementary Figure 1.

\section{Controlled Studies}

Ten of the 14 papers concerned RCTs. Having been published between 2008 and 2019, the papers examined 18 different mediators. Sample sizes varied from 46 to 440 participants, with an average sample size of 142.6 (SD 128.1). A total of 1,252 patients participated in the RCTs, with 638 in a BA condition and 614 in diverse control conditions. In the BA conditions, $78 \%$ of the patients were female compared with $79 \%$ in the control conditions ${ }^{1}$. Participants had average ages per study between 28.4 and 85.2 years. Common outcome measures of depression severity were the Beck Depression Inventory (BDI-II) [37] in 6 studies and the Patient Health Questionnaire (PHQ-9) [38] in 4 studies. Details about the reviewed studies can be found in online supplementary Table 1.

\section{Uncontrolled Studies}

The remaining 4 papers reported on non-randomised research and were published between 2008 and 2016 . They discussed 5 different mediators, 2 of which corresponded to the mediators in the included controlled studies. Sample sizes varied from 4 to 61 participants, with an average sample size of 23.5 (SD 26.4). A total of 94 patients participated in the uncontrolled studies, of whom 64.9\% were female. Participants had average ages per study ranging from 14.7 to 57.0 years. Two studies had a single-participant approach to mediation, while the other studies looked at mediation at the group level $[39,40]$.

\section{Quality Ratings}

An overview of the quality ratings for all studies can be found in online supplementary Table 2. Only 1 of the studies [4] fulfilled all quality requirements. Six studies included mediators that measured different competing

\footnotetext{
Based on 9 studies. One study (Armento et al. [43]) does not specify the sex of the patients in either condition.
} 
mechanisms in their analyses, but only 2 studies used them in the same model allowing their relative influence and specificity to be established $[4,41]$. Ten studies used an RCT design to test their mediation hypothesis. None of the studies used an experimental design, nor did any of the studies investigate a dose-response relationship between the mediators and treatment outcomes. Four studies used a temporal element to detect change in the mediators before change in outcome, but only 2 were RCTs $[4,42]$. Most studies used a representative BA protocol, although the intervention (BA) differed in duration from a single day to 3 months. One study [43] used a BA protocol that focused exclusively on religious behaviour, which deviates from the general approach of BA that focuses on a wide range of activities [44, 45].

\section{Main Results: The Mediators}

Activation

Of the 8 studies that used a mediator aimed at detecting changes in activation or "approach behaviour," only 2 demonstrated a mediation effect. The instrument most commonly used to assess this mediator was the Behavioural Activation for Depression Scale (BADS) [46]. The other scales that were used to measure activation or approach behaviour in the various studies are listed in online supplementary Table 1.

Three studies, with a total of 649 participants, examined the mediational effect of BADS on depression severity. Two studies (209 participants) found a mediational effect of activation in depressed pregnant women and adults with major depressive disorder (MDD), but only 1 of these studies could establish a (potentially causal) temporal relationship between improved BADS scores and reduced depression severity [42]. One study, with the largest number of participants $(n=440)$ and the highest quality score, did not find a mediation effect of activation on symptom severity of adult patients with MDD [4]. Using "engagement in pleasant activities" as a measure of activation, Ho [47] did not find a mediational effect in her treatment group of 49 patients. Rovner et al. [48] found that, except for social impairment, none of the BADS subscales mediated the relationship between treatment and depression outcome in their older patients with age-related macular degeneration, a condition that potentially causes a severe loss of vision.

\section{Environmental Reward}

Four studies focused on environmental reward as assessed with the Environmental Reward Observation Scale (EROS) [49]. The 2 studies with sufficient quality report- ed a significant mediational effect of EROS scores on depressive symptoms in pregnant women and women with breast cancer, respectively, but only the second study took temporality into account. $[42,50]$. In a secondary analysis of a subsample of the study by Hopko et al. [50], a mediating effect of environmental reward in the temporal relation between compliance and depression was not demonstrated [51]. One uncontrolled study demonstrated that environmental reward mediated the relationship between BADS and depressive symptoms in a sample of students with depressive symptoms. However, the authors did not investigate whether the observed change in BADS scores was caused by BA treatment [52].

Other Mediators

Significant findings were also reported for several other mediators, but most had only been investigated in single studies and were tailored to answer study-specific questions. Armento et al. [43] found a mediational effect of spiritual well-being in a version of BA specifically aimed at the modification of religious behaviours. In an RCT on BA for excessive worrying, Okajima and Chen [53] observed a mediational effect of insomnia on depression outcome. Two studies found more general mediators to have exerted significant effects. Studying the effect of BA and antidepressant medication, Moradveisi et al. [41] found that belief in self-coping skills did mediate the relationship between treatment and outcome, while belief in medication did not. Richards et al. [4] demonstrated mediation of treatment fidelity on 12-month follow-up depression scores, but only when it was the only mediator in the statistical model. Self-efficacy for controlling upsetting thoughts was a significant mediator in a study that investigated telephone-based BA for dementia caregivers [54]. None of the other studies examining whether cognitive factors such as rumination, dysfunctional thinking, and religious coping were mediators in BA treatment reported statistically significant effects $[4,43,53,55]$. Also, perceived social support (2 studies), anhedonia, anxiety, intolerance of uncertainty, work and social adjustment, and personal mastery did not mediate the relationship between BA and depression severity $[47,50]$.

\section{Discussion}

Below, we will summarise the main findings of our review and discuss the limitations of the various studies and our review process. More importantly, we will also dis-
88

Psychother Psychosom 2021;90:85-93 DOI: $10.1159 / 000509820$
Janssen/Hendriks/Baranelli/Lucassen/ Oude Voshaar/Spijker/Huibers 
cuss what these results, or the lack thereof, tell us about the way we need to shape studies in the future to really learn to understand the mechanisms of BA.

\section{Does Activation Make BA Effective?}

The mediational role of activation, as assessed with self-report scales, was not replicated consistently across studies and designs, rendering the strength of the evidence for this mediator weak. The study with the largest sample and highest quality score did not find a mediational effect of activation and, together with another nonsignificant study, accounted for almost $75 \%$ of the patients examined $[11,55]$. The 2 studies that did find statistically significant mediation effects were suboptimal, as one did not demonstrate a temporal path showing that the change in activation preceded improvement of depression [50], whereas the other study was limited to only 4 patients [48]. Furthermore, none of the studies applied an experimental design to test whether manipulation of the mediator directly affected changes in depression severity.

Is Environmental Reward the Key to Treatment Success?

All studies examining environmental reward found a positive mediating effect. However, to measure this they all used the EROS, which has a strong negative correlation with BDI-II (-0.80) [49], so no firm conclusions could be drawn. Armento and Hopko [49] argue that this correlation supports the convergent validity and the theoretical relationship between the diminished availability of environmental reward and depressive symptoms, but since the studies we reviewed did not substantiate that changes in environmental reward preceded the changes in depression, we cannot conclude whether they measured concurrent changes in environmental reward and depression or simply established symptom change with 2 different scales.

\section{Competing Theories}

Apart from environmental reward and activation, 19 other mediators were investigated. Of the mediators related to the cognitive model of depression [56] only selfefficacy for controlling upsetting thoughts [54] was found to be significant. Because of the pre-post design, and lack of specificity of mediators, it remains unknown whether improvements in self-efficacy can be seen as one of the mechanisms that explained the effect of BA on depression. Two studies $[4,48]$ found the BADS social impairment scale to account for the biggest part of the change in depression severity, but the effect was only significant in one [4]. The 2 studies that investigated social support as a mediator found no significant effects $[47,50]$. This justifies the conclusion that the strength of the evidence for social impairment and social support as mediators is low. Still, rather than social support, which is about the perceived actions of others, social impairment, i.e., a person's ability to seek social connection, might play a mediating role in BA and merits further investigation.

As most other mediators tested were non-significant or specifically selected to investigate purported mechanisms in specific study populations, they will not be discussed here further. Interestingly, however, when we look at 2 higher-quality studies $[5,8]$, the most effective mediators seem to be generic. Treatment fidelity mediated depression 12 months following BA completion [5], whereas belief in coping capabilities and crediting for self-improvement also mediated depression outcome [41]. Might BA be effective "merely" because people attend sessions and start believing in their own capabilities? Based on the results of our review, this question remains unanswered. At this stage, rather than just asking: "What mechanism should we investigate next?" we might also need to ask: "How can we best identify and quantify mediating mechanisms in BA?"

\section{Moving Forward from Here}

The fact that the reviewed studies - with substantial differences in quality - investigated 21 potential mediators in heterogeneous populations made it impossible to draw firm conclusions about any mediator of BA success. For future studies, we suggest to take into account the general recommendations for mediational research in psychotherapy provided by Lemmens et al. [29], such as using multiple sources of information and including a temporal design, as well our recommendations as stated below.

\section{Looking beyond the BA Theory}

Instead of focusing too heavily on existing theoretical models, it might be interesting to rely more on recent fundamental findings to sharpen definitions, to renew theoretical models, and generate new testable hypotheses. For example, in neurobiology the concept of anhedonia has been redefined as being "an interaction between reward motivation, liking, and learning" [57]. Studies have shown that the mesolimbic dopamine system, a brain region involved in reward processing and shown to have a reduced function in depressed patients [58], may be implicated in reward motivation but not in the experience of pleasure or "liking" of activities [59-62]. Experimental tasks in- 
deed revealed that depressed patients were less willing to work for rewards than healthy controls and that individual differences in the amount of high-effort choices made in such tasks are negatively associated with dopamine release in the insula $[63,64]$. In other words, patients coping with depression might underestimate the pleasure they will experience in relation to reward and consequently be less motivated to try to obtain it even though they might not like it any less than non-affected peers.

Building upon this knowledge, reward motivation, i.e., the ability to see the future as rewarding, might be a relevant potential mechanism of BA. Dichter et al. [24] used reward tasks to compare depressed people receiving BA to healthy controls. Unlike Treadway et al. [64], they did not find any behavioural differences between the 2 groups on the tasks themselves, while brain imaging suggested that BA appeared to normalise circuits related to reward motivation. Arguably, future research could be directed at uncovering whether reward motivation mediates treatment effects and employ brain imagery to further investigate whether changes in brain circuits related to motivational reward precede any intensification in the reward-anticipation and activation relationship.

\section{Tackling Temporality}

The 2 studies analysing mediation in single participants $[39,40]$ demonstrate that unravelling temporal relationships is challenging. These studies, classified as being of low quality, used an idiographic approach based on data obtained in individual patients rather than a nomothetic approach where group means are compared. This gives such studies an advantage that is not considered by Kazdin [26] because they expose the issue of causal heterogeneity, i.e., the phenomenon that conclusions are generalisable to an average group member but not to individual patients because of the large variability in individual pathways [65]. The studies indeed revealed that the pathways to improvement differed for the different patients $[39,40]$. Gaynor and Harris [40] found that in 2 of their 4 participants a change in activation preceded the change in depression. Folke et al. [39] observed that in some participants changes in approach behaviour preceded improvements in depression when measured daily, but when they looked at hourly ratings, changes in mood preceded the changes in activation.

The mediator-depression interaction is clearly a complex process which is unlikely to fit Kazdin's [26] model that includes one significant mediator, the change of which precedes the change in depression symptoms among several non-significant alternative mediators. Reality might be closer to Bandura's [66] concept of reciprocal determinism: the involvement of a reciprocal interaction between different influences, rather than a linear causal model, which determines psychological functioning. To get a better grasp of this interaction, it might be useful to start relying more on idiographic analyses. A way to do this might be the use of vector autoregressive (VAR) modelling to analyse individual pathways. VAR does not only reveal the temporal dynamics of different time series showing (potentially causal) relationships between mediators and depressive symptoms, but it also takes into account bidirectionality [65]. Furthermore, relying less on regular pre-set self-reporting but more on experience sampling (ESM), a research procedure in which individuals are asked to answer short questionnaires at random occasions during the day for a certain period of time [67], in addition to the use of an idiographic approach in bigger samples, will allow us to obtain data at multiple time points for each participant.

Bakker et al. [68] used ESM to ecologically validate the theory that depressed people are less sensitive to motivational reward. Their data showed that reward anticipation improved positive affect which then predicted active behaviour, but also that more activity enhanced positive affect, which, in turn, led to increased reward anticipation. These multidirectional pathways may explain why researchers are hard pressed to capture the process of change in a single model based on questionnaire scores obtained at fixed times. Another interesting, related topic for investigation would then be to see whether the relationship between reward anticipation and activity intensifies during BA and, if so, whether this strengthened relationship mediates treatment success, both at the group differences and the individual level.

\section{Limitations of the Current Review}

The present review has several limitations that should be acknowledged. Although we conducted an extensive search of the literature in 5 relevant databases and included both $\mathrm{PhD}$ theses and published research in our search, it is possible that some studies, particularly studies with non-English abstracts, were missed. Also, given the fact that the interactions between mediators and depression are likely to be non-linear, we might have been able to give a more complete view of those interactions if we had broadened our search to not only include depressive symptoms, but also other outcome measures such as wellbeing and social functioning. Furthermore, it has to be noted that the utilisation of our own tool to assess the quality of mediational studies makes a comparison between the included studies in this review and studies in other systematic reviews more difficult.
Janssen/Hendriks/Baranelli/Lucassen/ Oude Voshaar/Spijker/Huibers 


\section{Conclusion}

To the best of our knowledge, the current review is the first to systematically evaluate studies that investigated mediators of BA treatment for depression. Collectively, the reviewed studies demonstrate that the evidence for mediating mechanisms in BA is limited and that the process is too complex to explain with the designs used. This complexity should be addressed in future RCTs by including idiographic analyses as well as neurobiological parameters, in line with the National Institute of Mental Health experimental therapeutics approach [69].

\section{Acknowledgements}

We would like to thank On Ying Chan for her support with the database searches.

\section{Statement of Ethics}

This review was conducted in accordance with the Preferred Reporting Items for Systematic Reviews and Meta-Analyses (PRISMA) guidelines [30]. Methods and inclusion criteria were included in a protocol (PROSPERO registration: CRD42019119696) in advance of the study.

\section{Conflict of Interest Statement}

All authors declare that they have no conflicts of interest.

\section{Funding Sources}

This research did not receive any specific grant from funding agencies in the public, commercial, or not-for-profit sectors. Our ongoing RCT "Behavioural Activation by Mental Health Nurses for Late-Life Depression in Primary Care: A Randomized Controlled Trial" is funded by The Netherlands Ministry of Health Funding Programme for Health Care Efficiency Research ZonMw (843001606), as was the salary of the first author of the current systematic review. ZonMw had no involvement in the study design, in the collection, analysis, and interpretation of data, in the writing of the report, or in the decision to submit the current review for publication.

\section{Author Contributions}

Noortje P. Janssen: conceptualisation, methodology, investigation, writing - original draft, visualisation. Marcus J.H. Huibers: conceptualisation, supervision, methodology writing - reviewing and editing. Gert-Jan Hendriks: supervision, investigation, writing - reviewing and editing. Celine T. Baranelli: investigation. Peter Lucassen: supervision, writing - reviewing and editing. Jan Spijker: writing - reviewing and editing. Richard Oude Voshaar: writing reviewing and editing. All authors approved the final manuscript.

\section{References}

1 Luppa M, Sikorski C, Luck T, Ehreke L, Konnopka A, Wiese B, et al. Age- and gender-specific prevalence of depression in latest-life systematic review and meta-analysis. J Affect Disord. 2012 Feb;136(3):212-21.

2 Riedel-Heller SG, Busse A, Angermeyer MC. The state of mental health in old-age across the 'old' European Union- a systematic review. Acta Psychiatr Scand. 2006 May;113(5): 388-401.

3 World Health Organisation. Depression and other common mental disorders: global health estimates. Geneva: WHO; 2016.

4 Richards DA, Rhodes S, Ekers D, McMillan D, Taylor RS, Byford S, et al. Cost and Outcome of BehaviouRal Activation (COBRA): a randomised controlled trial of behavioural activation versus cognitive-behavioural therapy for depression. Health Technol Assess. 2017 Aug;21(46):1-366.

5 Dimidjian S, Hollon SD, Dobson KS, Schmaling $\mathrm{KB}$, Kohlenberg RJ, Addis ME, et al. Randomized trial of behavioral activation, cognitive therapy, and antidepressant medication in the acute treatment of adults with major depression. J Consult Clin Psychol. 2006 Aug;74(4):658-70.

6 Jacobson NS, Dobson KS, Truax PA, Addis ME, Koerner K, Gollan JK, et al. A component analysis of cognitive-behavioral treatment for depression. J Consult Clin Psychol. 1996 Apr; 64(2):295-304

7 Cuijpers P, van Straten A, Warmerdam L. Behavioral activation treatments of depression: a meta-analysis. Clin Psychol Rev. 2007 Apr; 27(3):318-26.

8 Ekers D, Webster L, Van Straten A, Cuijpers P, Richards D, Gilbody S. Behavioural activation for depression; an update of meta-analysis of effectiveness and sub group analysis. PLoS One. 2014 Jun;9(6):e100100.

9 Bolinski F, Hendriks G-J, Bardoel S, Hollon SD, Martell C, Huibers MJH. Cognitive therapy or behavioral activation for major depressive disorder in Dutch mental health care: pilot effectiveness and process trial. Int J Cogn Ther. 2018;11(3):343-58.

10 Ekers D, Richards D, McMillan D, Bland JM, Gilbody S. Behavioural activation delivered by the non-specialist: phase II randomised controlled trial. Br J Psychiatry. 2011 Jan; 198(1):66-72.

11 Ekers D, Godfrey C, Gilbody S, Parrott S, Richards DA, Hammond D, et al. Cost utility of behavioural activation delivered by the non-specialist. Br J Psychiatry. 2011 Dec; 199(6):510-1.

12 Dimidjian S, Barrera M Jr, Martell C, Muñoz RF, Lewinsohn PM. The origins and current status of behavioral activation treatments for depression. Annu Rev Clin Psychol. 2011;7(1):1-38.

13 MacPhillamy DJ, Lewinsohn PM. Depression as a function of levels of desired and obtained pleasure. J Abnorm Psychol. 1974 Dec;83(6):651-7.

14 Lewinsohn PM. A behavioral approach to depression. Essential papers on depression. New York: New York University Press; 1974. pp. 150-72.

15 Mazzucchelli T, Kane R, Rees C. Behavioral activation treatments for depression in adults: A meta-analysis and review. Clin Psychol Sci Pract. 2009;16(4):383-411.

16 Tindall L, Mikocka-Walus A, McMillan D, Wright B, Hewitt C, Gascoyne S. Is behavioural activation effective in the treatment of depression in young people? A systematic review and meta-analysis. Psychol Psychother. 2017 Dec;90(4):770-96.

17 Orgeta V, Brede J, Livingston G. Behavioural activation for depression for older people with or without cognitive impairment: a systematic review and meta-analysis of randomised controlled trials. Alzheimers Dement. 2017;13(7):499-500.

18 Cuijpers P, Reijnders M, Huibers MJ. The role of common factors in psychotherapy outcomes. Annu Rev Clin Psychol. 2019 May; 15(1):207-31. 
19 Grosscup SJ, Lewinsohn PM. Unpleasant and pleasant events, and mood. J Clin Psychol. 1980 Jan;36(1):252-9.

20 Lewinsohn PM, Graf M. Pleasant activities and depression. J Consult Clin Psychol. 1973 Oct;41(2):261-8.

21 Hopko DR, Armento ME, Cantu MS, Chambers LL, Lejuez CW. The use of daily diaries to assess the relations among mood state, overt behavior, and reward value of activities. Behav Res Ther. 2003 Oct;41(10):113748.

22 Hopko DR, Mullane CM. Exploring the relation of depression and overt behavior with daily diaries. Behav Res Ther. 2008 Sep;46(9): 1085-9.

23 Santos MM, Nagy GA, Hurtado GD, West P, Santiago-Rivera AL, Lee HJ, et al. Acculturative stress as a moderator of treatment engagement and retention in behavioral activation and treatment as usual for latinos with depression. J Lat Psychol. 2017 Jan;5(4):27589

24 Dichter GS, Felder JN, Petty C, Bizzell J, Ernst M, Smoski MJ. The effects of psychotherapy on neural responses to rewards in major depression. Biol Psychiatry. 2009 Nov;66(9): 886-97.

25 Pizzagalli DA, Jahn AL, O'Shea JP. Toward an objective characterization of an anhedonic phenotype: a signal-detection approach. Biol Psychiatry. 2005 Feb;57(4):319-27.

26 Kazdin AE. Mediators and mechanisms of change in psychotherapy research. Annu Rev Clin Psychol. 2007;3(1):1-27.

27 Kraemer HC, Wilson GT, Fairburn CG, Agras WS. Mediators and moderators of treatment effects in randomized clinical trials. Arch Gen Psychiatry. 2002 Oct;59(10):87783.

28 Kazdin AE. Understanding how and why psychotherapy leads to change. Psychother Res. 2009 Jul;19(4-5):418-28.

29 Lemmens LH, Müller VN, Arntz A, Huibers MJ. Mechanisms of change in psychotherapy for depression: an empirical update and evaluation of research aimed at identifying psychological mediators. Clin Psychol Rev. 2016 Dec;50:95-107.

30 Moher D, Liberati A, Tetzlaff J, Altman DG; PRISMA Group. Preferred reporting items for systematic reviews and meta-analyses: the PRISMA statement. Ann Intern Med. 2009 Aug;151(4):264-9.

31 Concato J, Horwitz RI. Limited usefulness of meta-analysis for informing patient care. Psychother Psychosom. 2019;88(5):257-62.

32 Guidi J, Brakemeier EL, Bockting CL, Cosci F, Cuijpers P, Jarrett RB, et al. Methodological recommendations for trials of psychological interventions. Psychother Psychosom. 2018; 87(5):276-84

33 MacKinnon DP, Fairchild AJ, Fritz MS. Mediation analysis. Annu Rev Psychol. 2007; 58(1):593-614

34 McGowan J, Sampson M, Lefebvre C. An evidence based checklist for the peer review of electronic search strategies. Evid Based Libr Inf Pract. 2010;5(1):149-54

35 Ouzzani M, Hammady H, Fedorowicz Z, Elmagarmid A. Rayyan-a web and mobile app for systematic reviews. Syst Rev. 2016 Dec; 5(1):210.

36 Cooper AA, Clifton EG, Feeny NC. An empirical review of potential mediators and mechanisms of prolonged exposure therapy. Clin Psychol Rev. 2017 Aug;56:106-21.

37 Beck AT, Steer RA, Brown GK. Beck depression inventory - II. San Antonio: Psychological Corporation; 1996. pp. 490-8.

38 Kroenke K, Spitzer RL. The phq-9: A new depression diagnostic and severity measure. Psychiatr Ann. 2002;32(9):509-15.

39 Folke F, Hursti T, Tungström S, Söderberg P, Kanter JW, Kuutmann K, et al. Behavioral activation in acute inpatient psychiatry: a multiple baseline evaluation. J Behav Ther Exp Psychiatry. 2015 Mar;46:170-81.

40 Gaynor ST, Harris A. Single-participant assessment of treatment mediators: strategy description and examples from a behavioral activation intervention for depressed adolescents. Behav Modif. 2008 May;32(3):372-402.

41 Moradveisi L, Huibers MJ, Arntz A. The influence of patients' attributions of the immediate effects of treatment of depression on long-term effectiveness of behavioural activation and antidepressant medication. Behav Res Ther. 2015 Jun;69:83-92.

42 Dimidjian S, Goodman SH, Sherwood NE, Simon GE, Ludman E, Gallop R, et al. A pragmatic randomized clinical trial of behavioral activation for depressed pregnant women. J Consult Clin Psychol. 2017 Jan; 85(1):26-36

43 Armento ME, McNulty JK, Hopko DR. Behavioral activation of religious behaviors (BARB): randomized trial with depressed college students. Psychol Relig Spiritual. 2012; 4(3):206-22.

44 Lejuez CW, Hopko DR, Hopko SD. A brief behavioral activation treatment for depression. Treatment manual. Behav Modif. 2001 Apr;25(2):255-86.

45 Martell CR, Addis ME, Jacobson NS. Depression in context: Strategies for guided action. New York: W.W. Norton \& Co; 2001.

46 Kanter J, Mulick P, Busch A, Berlin K, Martell C: Behavioral activation for depression scale (BADS)(long and short form). Galway: Measurement Instrument Database for the Social Sciences; 2012.

47 Ho JS. Mediators and moderators of dementia caregiver depression and CVD risk outcomes in the pleasant events program. UC San Diego Electron Theses Diss. 2016. https://escholarship.org/uc/item/4fw1417d.

48 Rovner BW, Casten RJ, Hegel MT, Massof RW, Leiby BE, Ho AC, et al. Low vision depression prevention trial in age-related macular degeneration: a randomized clinical trial. Ophthalmology. 2014 Nov;121(11):2204-11.

49 Armento ME, Hopko DR. The Environmental Reward Observation Scale (EROS): devel- opment, validity, and reliability. Behav Ther 2007 Jun;38(2):107-19.

50 Hopko DR, Clark CG, Cannity K, Bell JL. Pretreatment depression severity in breast cancer patients and its relation to treatment response to behavior therapy. Health Psychol. 2016 Jan; 35(1):10-8.

51 Ryba MM, Lejuez CW, Hopko DR. Behavioral activation for depressed breast cancer patients: the impact of therapeutic compliance and quantity of activities completed on symptom reduction. J Consult Clin Psychol. 2014 Apr;82(2):325-35.

52 Takagaki K, Okamoto Y, Jinnin R, Mori A, Nishiyama Y, Yamamura T, et al. Mechanisms of behavioral activation for late adolescents: positive reinforcement mediate the relationship between activation and depressive symptoms from pre-treatment to post-treatment. J Affect Disord. 2016 Nov;204:70-3.

53 Okajima I, Chen J. The effect of insomnia on changes in anxiety, depression, and social function after a transdiagnostic treatment targeting excessive worry. Sleep Biol Rhythms. 2017 Jan;15(3):243-9.

54 Au A, Yip HM, Lai S, Ngai S, Cheng ST, Losada A, et al. Telephone-based behavioral activation intervention for dementia family caregivers: outcomes and mediation effect of a randomized controlled trial. Patient Educ Couns. 2019;102(11):2049-59.

55 Nasrin F, Rimes K, Reinecke A, Rinck M, Barnhofer T. Effects of brief behavioural activation on approach and avoidance tendencies in acute depression: preliminary findings. Behav Cogn Psychother. 2017 Jan;45(1):58-72.

56 Beck AT, Weishaar M. Cognitive therapy; comprehensive handbook of cognitive therapy. Berlin: Springer; 1989. pp. 21-36.

57 Berridge KC, Robinson TE, Aldridge JW. Dissecting components of reward: 'liking', 'wanting', and learning. Curr Opin Pharmacol. 2009 Feb;9(1):65-73.

58 Nestler EJ, Carlezon WA Jr. The mesolimbic dopamine reward circuit in depression. Biol Psychiatry. 2006 Jun;59(12):1151-9.

59 Martin-Soelch C. Is depression associated with dysfunction of the central reward system? Biochem Soc Trans. 2009 Feb;37(Pt 1) 313-7.

60 Salamone JD. Functions of mesolimbic dopamine: changing concepts and shifting paradigms. Psychopharmacology. 2007 Apr; 191(3):389.

61 Bardgett ME, Depenbrock M, Downs N, Points M, Green L. Dopamine modulates effort-based decision making in rats. Behay Neurosci. 2009 Apr;123(2):242-51.

62 Berridge KC, Kringelbach ML. Affective neuroscience of pleasure: reward in humans and animals. Psychopharmacology. 2008 Aug; 199(3):457-80.

63 Treadway MT, Bossaller NA, Shelton RC, Zald DH. Effort-based decision-making in major depressive disorder: a translational model of motivational anhedonia. J Abnorm Psychol. 2012 Aug;121(3):553-8. 
64 Treadway MT, Buckholtz JW, Cowan RL, Woodward ND, Li R, Ansari MS, et al. Dopaminergic mechanisms of individual differences in human effort-based decision-making. J Neurosci. 2012 May;32(18):6170-6.

65 Rosmalen JG, Wenting AM, Roest AM, de Jonge $\mathrm{P}$, Bos EH. Revealing causal heterogeneity using time series analysis of ambulatory assessments: application to the association between depression and physical activity after myocardial infarction. Psychosom Med. 2012 May;74(4):377-86.

66 Bandura A. The self system in reciprocal determinism. Am Psychol. 1978;33(4):344-58.

67 Larson R, Csikszentmihalyi M. The experience sampling method: flow and the foundations of positive psychology. Berlin: Springer; 2014. pp. 21-34.
68 Bakker JM, Goossens L, Lange I, Michielse S, Schruers K, Lieverse R, et al. Real-life validation of reduced reward processing in emerging adults with depressive symptoms. J Abnorm Psychol. 2017 Aug;126(6):713-25.

69 Insel TR, Gogtay N. National Institute of Mental Health clinical trials: new opportunities, new expectations. JAMA Psychiatry. 2014 Jul;71(7):745-6. 